\title{
Study of attention measured by event- related potential as a predictive factor of violence among patients with schizophrenia and substance use disorder
}

\author{
Mohamed Elsheikh
}

\begin{abstract}
Background: The prediction of violence in patients with psychiatric disorders remains a challenging aspect in the field of clinical research. Many studies search the linkage between aggressive behavior and certain genetic conditions, impaired socio-emotional information processing, demographic, and clinical variables. To our knowledge, by far the relationship between aggressive behavior and impaired attention is not clear. Attention is one of the clinical variables that might have a relation to increased aggressive tendency in many psychiatric patients. So the purpose of this study is to measure the attention using ERP and search for its relation to violence in schizophrenic patients and patients with substance use disorder. This cross-sectional study was carried out on a sample of schizophrenic inpatients and patients with SUD (86 male patients) at the psychiatric department of AlHussein University Hospital, Cairo, Egypt.

Results: The majority of the sample lies in the age group 20-29years old (38.4\%), whereas MOAS showed that the higher tendency to violence was in the dual diagnosis of schizophrenia disorder with SUD followed by patients diagnosed with SUD.

- P300 wave measurement analysis reflected that the attention is highly affected in SUD patients, while schizophrenics showed delayed thinking. The relationship has a statistical significance $(P=0.001)$.

- A statistically significant direct correlation between P300 wave amplitude, latency, and the scale of violence was also found $(P=0.009$ and $P=0.022)$ respectively.
\end{abstract}

Conclusion: Affected attention in both schizophrenic and SUD patients could be considered a potential risk factor of violence.

Keywords: Attention, Event-related potential, P300, Violence, SUD, Schizophrenia

\section{Background}

The prediction of violence in psychiatric patient populations remains one of the most challenging aspects of work with psychiatric patients [1].

Accurate assessment depends on the availability of accurate information. This will usually include information

Correspondence: mohamed.elscheikh@gmail.com

Department of Psychiatry, Faculty of Medicine, Al-Azhar University, Cairo, Egypt obtained from collateral sources such as medical records, informants or police reports. However, the clinical assessment carried out on admission has limited power in assessing the predictors of potential violence among psychiatric patients. Therefore, additional investigations, including psychological testing, and measuring attention, may be required.

Previous research has linked aggressive behavior to certain genetic conditions, impaired socio-emotional

\section{Springer Open}

(c) The Author(s). 2020 Open Access This article is licensed under a Creative Commons Attribution 4.0 International License, which permits use, sharing, adaptation, distribution and reproduction in any medium or format, as long as you give appropriate credit to the original author(s) and the source, provide a link to the Creative Commons licence, and indicate if changes were made. The images or other third party material in this article are included in the article's Creative Commons licence, unless indicated otherwise in a credit line to the material. If material is not included in the article's Creative Commons licence and your intended use is not permitted by statutory regulation or exceeds the permitted use, you will need to obtain permission directly from the copyright holder. To view a copy of this licence, visit http://creativecommons.org/licenses/by/4.0/. 
information processing, demographic variables (as gender and age) and clinical variables (as diagnosis, presence of mood symptoms, and comorbid substance abuse). An association between aggression and inherent cognitive defects-such as impaired information processing, socio-emotional understanding, and problemsolving skills-has been demonstrated in patients with mental retardation and schizophrenia. Inaccuracy in correctly identifying interpersonal intent, a tendency wrongly to attribute hostile intent to others, as well as a poorer ability to assess the intensity of emotion has also been demonstrated [2].

On the other hand, the predictors of violence depend on the setting: whereas clinical and psychopathological variables may predict violence in institutional settings, demographic and historical variables are better predictors in community settings and in clinical samples consisting of only high-risk patients.

The complex influence of diagnosis on psychiatric patients' risk of violence has emerged from a variety of studies in different contexts. First, a diagnosis of schizophrenia (and other severe, enduring psychotic disorders) has been demonstrated unequivocally to increase a person's risk of violence in comparison to the general population [3].

Similarly, comorbid substance abuse/dependence dramatically increases the risk of violence in patients with schizophrenia [4].

Primary diagnosis of substance abuse/dependence has also been identified as a strong predictor of violence in psychiatric patients [5].

Another factor that appears to elevate the risk of violence in patients with schizophrenia is the presence of neurological damage, e.g., parieto-occipital atrophy, reduced grey matter volume in neural circuits involved in verbal working memory, as well as temporal EEG abnormalities [6]. However, a diagnosis of epilepsy itself has not been proven to increase the risk of violence [7].

Comorbid substance abuse increases the risk of violence in patients with mental retardation. Furthermore, violent mentally retarded adults have been shown to have larger brain ventricles than their non-violent counterparts, as well as a higher frequency of abnormal EEGs, yet no increased prevalence of seizure disorders (as for schizophrenia) [8].

This aggressive diathesis can be conceptualized in terms of an imbalance between the "top-down" control or "brakes" provided by the orbital frontal cortex and anterior cingulate cortex, which are involved in calibration of behavior to social cues and predicting expectancies of reward and punishment, and thus modulating or suppressing aggressive behavior with negative consequences, and excessive "bottom-up" "drives" triggered or signaled by limbic regions, such as the amygdala and insula. An emotionally provocative or challenging stimulus that serves as a trigger to the aggressive event will initially be processed by auditory, visual, and other sensory processing centers. At this stage, sensory deficits such as hearing or visual impairment as well as sensory distortions that might be caused by drugs, alcohol, or metabolic disturbances secondary to illness may result in incomplete or distorted sensory impressions, which can increase the likelihood that the stimulus is perceived as threatening or provocative [9].

Attention is the behavioral and cognitive process of selectively concentrating on a discrete aspect of information, whether subjective or objective, while ignoring other perceivable information. Attention has also been referred to as the allocation of limited processing resources; it is best described as the sustained focus of cognitive resources on information while filtering or ignoring extraneous information. Attention is a very basic function that often is a precursor to all other neurological/cognitive functions [10].

Hence, its affection may be related to different abnormal behaviors including increased violence tendency. The event-related potential (ERP) is the measured brain response that is the direct result of a specific sensory, cognitive, or motor event. More formally, it is any stereotyped electrophysiological response to a stimulus. The study of the brain in this way provides a noninvasive means of evaluating brain functioning including attention. ERPs are measured by means of electroencephalography (EEG).

The EEG proved to be a useful source in recording brain activity over the ensuing decades. However, it tended to be very difficult to assess the highly specific neural processes that are the focus of cognitive neuroscience because using pure EEG data made it difficult to isolate individual neurocognitive processes. Event-related potentials (ERPs) offered a more sophisticated method of extracting more specific sensory, cognitive, and motor events by using simple averaging techniques. Currently, ERP is one of the most widely used methods in cognitive neuroscience research to study the physiological correlates of sensory, perceptual, and cognitive activity associated with processing information.

The P300 (P3) wave is an event-related potential (ERP) component elicited in the process of decision-making. It is considered to be an endogenous potential, as its occurrence links not to the physical attributes of a stimulus, but to a person's reaction to it. More specifically, the P300 is thought to reflect processes involved in stimulus evaluation or categorization. It is usually elicited using the oddball paradigm, in which low-probability target items are mixed with high-probability nontarget (or "standard") items [11]. 
The P300 response occurs at around $300 \mathrm{~ms}$ in the oddball paradigm, for example, regardless of the type of stimulus presented: visual, tactile, auditory, olfactory, and gustatory.

Because of this general invariance with regard to stimulus type, the P300 component is understood to reflect a higher cognitive response to unexpected and/or cognitively salient stimuli [12].

\section{Methods}

\section{Aim of study}

The aim of this study is to measure the attention using ERP and its relation to violence in schizophrenic patients and patients with substance use disorder (SUD).

\section{Study design}

This is a cross-sectional descriptive study.

\section{Site of the study and selection of participants}

The study was conducted at the psychiatric inpatient department of Al-Hussien University Hospital in Cairo on a sample of patients diagnosed with schizophrenia and substance use disorder (SUD) who met the inclusion criteria. The patients who participated in the study were all males, as the criteria of admission to the addiction unit at Al-Hussien University Hospital were restricted to male patients. Therefore, recruiting female patients with SUD was not possible and hence there would not have been a SUD counterpart for the female schizophrenic patients.

During the period of the study that lasted from August 2013 until July 2014, the total number of male inpatients diagnosed with schizophrenia and/or SUD was 143 patients. Among them, twenty-three patients refused to participate in the study, while two patients were excluded after confirming a comorbid endocrine disorder and lupus erythematosus. Also, thirty-two patients who showed neurological deficits, whether clinically or through EEG changes and thrombotic foci in the MRI, were excluded from the study. Thus, we were left with 86 patients (38 schizophrenics, 32 patients with SUD and 16 with a dual 51 diagnosis).

\section{Inclusion criteria}

1. Male patients in the age group of 18 to 65 years

2. Diagnosed with schizophrenia or substance use disorder (SUD) according to DSM-IV-TR

3. Agreed to participate in the study by giving explicit written and verbal consent

\section{Exclusion criteria}

1. Presence of any organic or neurological comorbidity/ deficits that might interfere with the results of investigation
2. Existence of problem that makes communication difficult such as hearing impairment or inability to talk

3. Refusal to participate in the study

\section{Ethical considerations and approvals}

A written informed consent was taken from each participant to whom we explained the full procedure and the aim of the study. All the participants had the right to withdraw from the research at any time without giving reasons, and without any negative consequences.

\section{Tools}

1. Structured Clinical Interview for DSM-IV-Clinician Version (SCID-CV) [13] Arabic version [14]. It contains seven diagnostic modules. The clinician version was used for easier application in the clinical setting.

2. Assessment for violence tendency using the modified overt aggression scale (MOAS)

The modified overt aggression scale (MOAS) was developed to assess four types of aggressive behavior: verbal aggression, aggression against property, auto aggression, and physical aggression. The MOAS instructs the individual (nursing staff, physicians, or medical trainees) to rate the patient's aggressive behaviors over the past week.

The MOAS was applied by the attending medical and nursing staff. The MOAS is individually administered and generally administered by nursing staff as no specific qualifications are required [15].

3. Measurement of attention using event-related potentials mainly P300 wave

4. Statistical analysis: data were fed to the computer and analyzed using the IBM SPSS software package version 20.0 [16]. Qualitative data were described using number and percent. Quantitative data were described using range (minimum and maximum), mean, standard deviation, and median. Comparison between different groups regarding categorical variables was tested using chi-square test. The distributions of quantitative variables were tested for normality using KolmogorovSmirnov test, Shapiro-Wilk test, and D'Agstino test.

( $r$ ) Pearson Correlation coefficient was used to determine correlation between relationships. The characteristics of the Pearson correlation coefficient are as follows: (1) The correlation scale determines the relationship between only two variables. (2) The correlation coefficient value is always between -1 and 1.3. If the correlation coefficient is positive, the correlation is direct. This means that the value of the first variable increases the value of the second variable. (4) If the correlation coefficient is negative, the correlation is inverse. That is, the 
increase in the value of the first variable leads to the decline of the value of the second variable. (5) The correlation is very strong when its value approaches 1 or - 1.6. Approaching zero means a weak relationship. If the correlation value is zero, the relationship between the two variables is zero.

\section{Results}

In this study, the age group 20-29 is the most representative group with $38.4 \%$, as shown in Table 1 , which demonstrates demographic characteristics. The majority of participants were single $73.3 \%$, non-educated $59.3 \%$, and unemployed $73.3 \%$ of the sample.

While Table 2 simplifies the relation between MOAS (modified overt aggression scale) scores calculated in mean and demographic data of the sample, showing that the highest score of aggression occurred in age group $(18-<20$ years) (mean $19.0 \pm$ SD 12.07), it also shows that the score is higher in the married, high educational level, and in working groups than the other participants. The group of participants with dual diagnosis was the

Table 1 Sample distribution according to demographic data $(n=86)$

No.

Percent

Age (by years)

\begin{tabular}{|c|c|c|}
\hline $18-<20$ & 6 & $7.0 \%$ \\
\hline $20-29$ & 33 & $38.4 \%$ \\
\hline $30-39$ & 26 & $30.2 \%$ \\
\hline $40-49$ & 16 & $18.6 \%$ \\
\hline $50-59$ & 5 & $5.8 \%$ \\
\hline \multicolumn{3}{|l|}{ larital status } \\
\hline Single & 63 & $73.3 \%$ \\
\hline Married & 23 & $26.7 \%$ \\
\hline \multicolumn{3}{|l|}{ ducational level } \\
\hline High & 5 & $5.8 \%$ \\
\hline Middle & 30 & $34.9 \%$ \\
\hline Illiterate & 51 & $59.3 \%$ \\
\hline \multicolumn{3}{|l|}{ Vorking } \\
\hline No work & 63 & $73.3 \%$ \\
\hline Working & 23 & $26.7 \%$ \\
\hline \multicolumn{3}{|l|}{ ype of work } \\
\hline No work & 37 & $43.0 \%$ \\
\hline Manual worker & 39 & $45.3 \%$ \\
\hline Clerical work & 2 & $2.3 \%$ \\
\hline Driver & 3 & $3.5 \%$ \\
\hline Accountant & 3 & $3.5 \%$ \\
\hline Lawyer & 1 & $1.2 \%$ \\
\hline Teacher & 1 & $1.2 \%$ \\
\hline
\end{tabular}

highest to record violence tendency on MOAS (mean $33.13 \pm$ SD 5.66) as shown in Table 3.

Table 4 shows the relationship between the substance use and the violence scale. The tendency of violence in the group of SUD patients was four times higher than the rest of the sample (mean 22.94 \pm SD 10.81).

In addition, the value of P300 wave amplitude and latency varied among the three participant groups as demonstrated in Table 5. The P300 amplitude recorded higher values in schizophrenic patients' group (mean $36.34 \pm$ SD 11.7) than the other two participant groups. Unlike P300 amplitude, P300 latency recorded the shorter latency in the SUD group (mean $367.05 \pm$ SD 80.98 ), which significantly concludes that the attention is highly affected in patients with SUD $(P$ value $=0.001)$ while schizophrenic patients show delayed thinking $(P$ value $=0.001$ ).

A direct correlation between P300 wave amplitude and latency and the scale of violence was made using Spearman coefficient as shown in Tables 6 and 7 with $P$ value of $(0.009$ and 0.022$)$ respectively.

\section{Discussion}

Previous studies examining the relationship between violence and psychiatric disorders were conducted in one of three ways: evaluation of violence in psychiatric patients, or diagnosis of psychiatric disorders in people who committed acts of violence, or evaluation of violence in psychiatric and other non-psychiatric patients [17]. Our current study is a trial to figure out the role of affected attention in increased tendency to violence among schizophrenic patients and patients with SUD. In addition, we compared the incidence of violence among different patient groups and their variable demographic determinants. The demographic distribution of the sample came in line with most of the research studies concerned with SUD and schizophrenia. In our study, 38\% are in the age group 20-29 years, which resembles the results of the national research of addiction in Egypt [18], where $37.9 \%$ of the participants were $20-29$ years. This age group is the most representative age group of substance use problems worldwide, as well as for schizophrenia requiring hospitalization [19]. This is also consistent with the prevalence of schizophrenia between 20 and 29 years [20]. Being a younger age is a risk factor for increasing the tendency to violence supported by a great body of research stating the hypothesis of increased impulsivity in the adolescence more than in other age groups.

Single patients represent $73.3 \%$ of the sample, which is the largest group represented in the sample. This may be explained first by the younger age of the majority of our sample 20-29 years. Secondly, both schizophrenia 
Table 2 Relation between the scale of violence (modified overt aggression scale) and demographic data $(n=86)$

\begin{tabular}{|c|c|c|c|c|c|c|}
\hline & \multirow[t]{2}{*}{$\mathbf{N}$} & \multicolumn{3}{|c|}{ Modified overt aggression scale } & \multirow{2}{*}{$\begin{array}{l}\text { Test of } \\
\text { sig. }\end{array}$} & \multirow[t]{2}{*}{$P$} \\
\hline & & Min.-max. & Mean \pm SD & Median & & \\
\hline \multicolumn{7}{|l|}{ Age (years) } \\
\hline $18-<20$ & 6 & $3.0-30.0$ & $19.0 \pm 12.07$ & 23.0 & $H=1.799$ & 0.773 \\
\hline $20-29$ & 33 & $0.0-40.0$ & $14.52 \pm 12.90$ & 11.0 & & \\
\hline $30-39$ & 26 & $0.0-39.0$ & $13.54 \pm 10.74$ & 9.50 & & \\
\hline $40-49$ & 16 & $0.0-40.0$ & $17.06 \pm 14.21$ & 12.50 & & \\
\hline $50-59$ & 5 & $7.0-35.0$ & $17.20 \pm 10.87$ & 14.0 & & \\
\hline \multicolumn{7}{|c|}{ Marital status } \\
\hline Single & 63 & $0.0-40.0$ & $14.30 \pm 12.65$ & 9.0 & $U=576.0$ & 0.147 \\
\hline Married & 23 & $0.0-40.0$ & $17.52 \pm 10.85$ & 17.0 & & \\
\hline \multicolumn{7}{|c|}{ Educational level } \\
\hline High & 5 & $6.0-37.0$ & $22.20 \pm 11.65$ & 23.0 & $H=2.273$ & 0.321 \\
\hline Moderate & 30 & $0.0-35.0$ & $15.50 \pm 11.12$ & 13.0 & & \\
\hline Illiterate & 51 & $0.0-40.0$ & $14.27 \pm 12.86$ & 9.0 & & \\
\hline \multicolumn{7}{|l|}{ Work } \\
\hline No & 63 & $0.0-40.0$ & $14.92 \pm 12.71$ & 10.0 & $U=655.50$ & 0.500 \\
\hline Yes & 23 & $0.0-37.0$ & $15.83 \pm 10.97$ & 13.0 & & \\
\hline
\end{tabular}

$U$ Mann Whitney test, $\mathrm{HH}$ for Kruskal Wallis test, $P$ P value for comparing association between modified overt aggression scale and demographic data *Statistically significant at $P \leq 0.05$

and SUD noticeably affect the social functions of patients rendering them unmarried or separated [21].

Regarding the level of education, the non-educated category was more represented in the sample 59.3\%, followed by the average education $34.9 \%$. The majority of the sample did not work at the time of conducting the study accounting for $73.3 \%$ of the sample. This corresponds to the Swanson's study investigating violence in patients with severe mental illness, where a sample of 804 patients received inpatient and outpatient treatment in four states where most of the participants were illiterate and not working [22]. Our findings may be explained additionally by the fact that the sample was taken from Al-Hussein University Hospital, which serves to a major extent to a certain class of patients with low socio-economic states from Al-Manshia and Al-Dweka.
Married patients living together with their spouses recorded higher levels of violence than single participants, as they have more friction points and conflict potential in their home environment than single participants. Similarly, violence was noticed more in working participants at work places than in non-working participants.

Unexpectedly, violence was recorded more in highly educated participants which may be explained by increased sensitivity to aggressive potentials, while other groups of participants may trivialize these aggressive potentials or interpreting these as normal acts in their environment.

By analysis, the relation between modified overt aggression scale and psychiatric disorders in the sample to assess the violence in Tables 3 and 4, the highest rate of violence was seen in SUD patients, nearly four times more than other participants.

Table 3 Distribution of the sample according to the relationship between the scale of violence and psychiatric disorders

\begin{tabular}{lllll}
\hline & SUD pt. $(\boldsymbol{n}=\mathbf{3 2})$ & Dual diagnosis pt. $(\boldsymbol{n}=\mathbf{1 6})$ & Schizophrenia pt. $(\boldsymbol{n}=\mathbf{3 8})$ & ${ }^{\mathrm{Kw}} \mathbf{X}^{\mathbf{2}}$ \\
\hline MOAS & & & $\boldsymbol{P}$ \\
Min.-max. & $5.0-37.0$ & $26.0-40.0$ & $0.0-15.0$ & $55.300^{*}$ \\
Mean \pm SD & $17.84 \pm 9.02$ & $33.13 \pm 5.66$ & $5.39 \pm 4.55$ & $<0.001^{*}$ \\
Median & 18.0 & 32.50 & 5.50
\end{tabular}

Sig. bet. grop.

$P_{1}<0.001^{*}, P_{2}<0.001^{*}, P_{3}<0.001^{*}$

$K W$ chi-square test value for Kruskal Wallis test, $P_{1} P$ value for Mann Whitney test for comparing between SUD pt. and dual diagnosis pt., $P_{2} P$ value for Mann Whitney test for comparing between SUD pt. and schizophrenia pt., $P_{3} P$ value for Mann Whitney test for comparing between dual diagnosis pt. and schizophrenia pt.

*Statistically significant at $P \leq 0.05$ 
Table 4 Sample distribution according to the relationship between violence and substance use disorder

\begin{tabular}{lllll}
\hline & $\begin{array}{l}\text { Non SUD pt. } \\
(\boldsymbol{n}=\mathbf{3 8})\end{array}$ & $\begin{array}{l}\text { SUD pt. } \\
(\boldsymbol{n}=\mathbf{4 8})\end{array}$ & $\boldsymbol{Z}$ & $\boldsymbol{P}$ \\
\hline MOAS & & & & \\
Min.-max. & $0.0-15.0$ & $5.0-40.0$ & $6.775^{*}$ & $<0.001^{*}$ \\
Mean. \pm SD & $5.39 \pm 4.55$ & $22.94 \pm 10.81$ & & \\
Median & 5.50 & 23.0 & & \\
\hline
\end{tabular}

$Z Z$ for Mann Whitney test

*Statistically significant at $\underline{P} \leq 0.05$

This may be attributed to the impulsive personality traits of most of substance abusing persons, as concluded by [23] in his study on patients with combined SUD and antisocial personality disorder (ASPD). Moreover, craving for substance is another factor contributes in increasing violence tendency, as this may urge the individual to do a violent act to obtain the substance. In addition, the rational behavior and judgment are mostly altered under the toxic effect of the substance, making individual more susceptible to act out the aggressive impulses unlike non-addict people due to poor decisionmaking as stated by Zois and his colleagues at the central institute of mental health in Mannheim, Germany, who have studied the decision-making deficits in patients diagnosed with disordered gambling (DG) with or without SUD, and they concluded that patients with DG with or without SUD not only having riskier decisionmaking profile but also are liable to take more risks rendering them more susceptible to violence [24].

The highest levels of violence appeared in patients with a dual diagnosis as shown in Table 3 followed by the group of SUD patients and the relationship are statistically significant. This came in accordance with the
Table 6 The correlation between the violence scale and P300 wave amplitude

\begin{tabular}{lll}
\hline & \multicolumn{2}{l}{ Modified overt aggression scale } \\
\cline { 2 - 3 } & $r$ Spearman coefficient & $P$ statistical sig. \\
\hline P300 wave amplitude & $0.281^{*}$ & $0.009^{*}$ \\
\hline
\end{tabular}

results of Janis Kelly's study, where it was assumed that the rate of violence increased in dual diagnosis patients having schizophrenia and SUD concurrently by 4 to 6 times than non-addictive patients. The researcher studied the records of schizophrenic patients who were admitted in psychiatric hospitals in Sweden and criminal convictions from 1973 to 2006 and after studying the different factors of age, sex, economic level, and drug use. The researcher also found that the increase in violence crimes in mental ill patients is the result of a double diagnosis of dependence on drugs, with the rate of violence four times more dependent on drug dependence than others [25]. The rate of violence is five to six times higher when comparing dual diagnosis patients with schizophrenic patients alone, while the violence doubles three to four times when comparing drug dependence and schizophrenic patients.

Unlike schizophrenia, substance use is a major cause of increased rates of violence, as it is characterized by increased impulsivity and affection of attention to a degree that affects anger control, and even makes patients unaware of their behavior consequences. This is consistent with some of the results in Fazel's study as it was a comprehensive study looking at the databases and checklists registered for mental patients who have done any violence and analyzed the data and found that it increases the rates of violence if involved with the disorder of

Table 5 The relationship between psychiatric disorders and P300 wave amplitude and latency

\begin{tabular}{|c|c|c|c|c|c|}
\hline & SUD pt. $(n=32)$ & Dual diagnosis pt. $(n=16)$ & Schizophrenia pt. $(n=38)$ & ${ }^{k W} x^{2}$ & $P$ \\
\hline \multicolumn{6}{|l|}{ P300 amplitude } \\
\hline Min.-max. & $2.90-51.0$ & $7.90-40.20$ & $11.80-65.30$ & $29.415^{*}$ & $<0.001^{*}$ \\
\hline Mean \pm SD & $18.18 \pm 12.91$ & $23.39 \pm 9.72$ & $36.34 \pm 11.74$ & & \\
\hline Median & 14.25 & 21.20 & 37.55 & & \\
\hline Sig. bet. grop. & \multicolumn{5}{|c|}{$P_{1}=0.052, P_{2}<0.001^{*}, P_{3}<0.001^{*}$} \\
\hline \multicolumn{6}{|l|}{ P300 latency } \\
\hline Min.-max. & $232.23-553.35$ & $263.80-923.10$ & $156.90-825.30$ & $38.637^{*}$ & $<0.001^{*}$ \\
\hline Mean \pm SD & $367.05 \pm 80.98$ & $650.18 \pm 158.02$ & $658.06 \pm 175.0$ & & \\
\hline Median & 357.55 & 667.05 & 699.65 & & \\
\hline Sig. bet. grop. & \multicolumn{5}{|c|}{$P_{10}<0.001^{*}, P_{20}<0.001^{*}, P_{30}=0.384$} \\
\hline
\end{tabular}

$K W$ chi-square test value for Kruskal Wallis test, $P_{1} P$ value for Mann Whitney test for comparing between SUD pt. and dual diagnosis pt., $P_{2} P$ value for Mann Whitney test for comparing between SUD pt. and schizophrenia pt., $P_{3} P$ value for Mann Whitney test for comparing between dual diagnosis pt. and schizophrenia pt., $P_{10} P$ value for Mann Whitney test for comparing between SUD pt. and dual diagnosis pt., $P_{20} P$ value for Mann Whitney test for comparing between SUD pt. and schizophrenia pt., $P_{30} P$ value for Mann Whitney test for comparing between dual diagnosis pt. and schizophrenia pt.

*Statistically significant at $P \leq 0.05$ 
Table 7 The correlation between the violence scale and P300 wave latency

\begin{tabular}{lll}
\hline & \multicolumn{2}{l}{ Modified overt aggression scale } \\
\cline { 2 - 3 } & $r$ Spearman coefficient & $\boldsymbol{P}$ statistical sig. \\
\hline P300 wave latency & $0.274^{*}$ & 0.022 \\
\hline
\end{tabular}

*Statistically significant at $P \leq 0.05$

dependence on drugs, As they indicate that the increase in the rate of violence is equal if the diagnosis is double or drug dependence disorder [26]. It also agrees with Witt's study of violence in schizophrenic patients and its increase with complicity in drug use [27]. These results may differ from the study of Short, where he studied the behaviors of violence in mental ill patients after discharge from the hospital, and a random sample of the community and a group of psychotic patients share the dependence on drugs and concluded that the study increased the rate of violence in mental ill patients compared to the sample where both mental patients alone or double dependence on drugs. The difference may be explained by the fact that the study of acts or behaviors of violence after they have been discharged from the hospital is in the recovery phase [28].

In our study, attention measured by ERP was highly affected in all sample subgroups in different ways as follows: by analysis of P300 amplitude, the lowest amplitude was seen in SUD group, while the highest values were seen in the schizophrenic group, and the relation was statistically significant. And by analysis P300 wave latency, the longer latency was in schizophrenic group.

These results show that attention has variably affected as the wave amplitude indicates the temporal factor for response (occurrence of target in relation to the stimulus) [29]. And as Wave amplitude is considered an indicator of the ability to pay attention to a person's action, which is strongly affected in SUD patients [30]. This may explain that addiction adversely affects wave amplitude as it decreases with substance abuse.

This is consistent with Brian's study that investigated the impact of external factors on attention such as substance abuse on the amplitude of P300 wave, the research based the study of the P300 wave amplitude in the twins, some of them were identical and other asymmetric and found that drug dependence disorder reduces the amplitude (Hicks, et al. 2007).

And as P300 wave latency refers to slow thinking and slower information processing, the results explain that schizophrenia significantly increases the latency of the wave. This is also consistent with Qiu results who found a delay of the wave P300 (increase in latency) in schizophrenic patients than in healthy controls [31]. It also agrees with the Simlai's study, which showed that schizophrenia disrupts the latency of the wave [32].
The effect on both P300 wave amplitude and latency indicates a lack of brain activity, and subsequently affection of attention [33].

The relation between violence measured by MOAS and attention measured by P300 wave amplitude and latency in this study found that both the wave amplitude and latency can be considered as determinants of violence. Pearson correlation coefficient $(r)$ shown in Tables 6 and 7 has been used for this purpose and showed a positive weak correlation. However, with weak correlation, the relationship in the two determinants has a statistical significance with $P$ value of $(0.009$ and 0.022$)$ respectively. This statistical analysis indicates that affection of attention as detected by P300 should be considered determinant risk factors for violence.

\section{Limitations}

The whole sample was exclusively recruited from a male ward, so that female gender was not represented in this study. Moreover, the number of participants was not big enough.

\section{Conclusion}

The current study concluded that:

- One of determinant risk factors for violence is the affection of attention as detected by ERP mainly P300 wave amplitude and latency.

- There is a weak direct correlation between the occurrence of violence MOAS and P300 wave amplitude and latency.

- Both SUD and schizophrenia are found to affect attention.

- The level of violence increases in patients with substance abuse as dual diagnosis with schizophrenia more than those with schizophrenia alone. So substance abuse itself considered as determinant for violence.

- The level of violence in patients with schizophrenia increases sharply if there is dual diagnosis with SUD.

\section{Abbreviations}

MOAS: Modified overt aggression scale; P300: Deflection emerges in a timelocked record as a positivity typically appearing approximately 300 to $400 \mathrm{~ms}$ following stimulus presentation; SCID I: The Structured Clinical Interview for DSM-IV Axis I Disorders

\section{Acknowledgements}

Not applicable

The committee's reference number N/A

Authors' contributions

ME analyzed and interpreted the patients' data, and was the contributor in writing the manuscript. The author read and approved the final manuscript. 


\section{Funding}

None.

\section{Availability of data and materials}

Data sharing is not applicable to this article as no datasets were generated or analyzed during the current study.

\section{Ethics approval and consent to participate}

The approval of the patients is confirmed orally and in writing according to the standards of the Ethics Committee at the Faculty of Medicine, Al-Azhar University, and to familiarize them with the study methodology and their right to withdraw at any time.

\section{Consent for publication Not applicable}

\section{Competing interests}

None declared

Received: 15 October 2019 Accepted: 24 February 2020

Published online: 08 May 2020

\section{References}

1. Elbogen EB, Johnson SC (2009) The intricate link between violence and mental disorder: results from the National Epidemiologic Survey on Alcohol and Related Conditions. Archives of General Psychiatry 2009 66(2):152-161

2. Silver H, Goodman C, Knoll G, Isakov V, Modai I (2005) Schizophrenia patients with a history of severe violence differ from nonviolent schizophrenia patients in perception of emotions but not cognitive function. Journal of Clinical Psychiatry 2005 66(3):300-308

3. Soyka M, Graz C, Bottlender R, Dirschedl P, Schoech H (2007) Clinical correlates of later violence and criminal offences in schizophrenia. Schizophrenia Research 2007 94(1-3):89-98

4. Swanson JW, Swartz MS, Van Dorn RA, Elbogen EB, Wagner HR, Rosenheck RA, et al.(2006) A national study of violent behavior in persons with schizophrenia. Archives of General Psychiatry 2006; 63(5):4909.

5. Lunsky Y, Bradley EA, Gracey CD, Durbin J, Koegl C (2009) Gender differences in psychiatric diagnoses among inpatients with and without intellectual disabilities. American Journal on Intellectual \& Developmental Disabilities 2009 114(1):52-60

6. Joyal CC, Gendron C, Cote G (2008) Nature and frequency of aggressive behaviours among long-term inpatients with schizophrenia: a 6 month report using the modified overt aggression scale. Canadian Journal of Psychiatry 2008 53(7):478-481

7. Puri BK, Counsell SJ, Saeed N, Bustos MG, Treasaden IH, Bydder GM.(2008) Regional grey matter volumetric changes in forensic schizophrenia patients: an MRI study comparing the brain structure of patients who have seriously and violently offended with that of patients who have not. BMC Psychiatry 2008; 8(Suppl 1):S6.

8. May PB Jr, DeMarco K, London EB, Thompson R, Mento TL, Buscemi L, Cody $R$ (2001) Ventricular enlargement in adults with profound mental retardation who demonstrate violent/destructive behaviors. Journal of Neuropsychiatry \& Clinical Neurosciences 2001 3(1):96-100

9. Best M, Williams JM, Coccaro EF (2002) Evidence for a dysfunctional prefrontal circuit in patients with an impulsive aggressive disorder. Proc Natl Acad Sci U S A 99:8448-8453

10. Johnson A. Proctor R. (2004). Attention: theory and practice. Liberary of congress cataloging-in-publication data. ISBN: 0-7619-2760-

11. Polich J (2007) Updating P300: an integrative theory of P3a and P3b. Clinical Neurophysiology 118(10):2128-2148

12. Todd C. Handy (2005). Event related potentials: a methods handbook. A Bradford book press. ISBN 0-262-08333-7.

13. First MB, Spitzer RL, Gibbon M, Williams JBW, Benjamin LS (1997) Structured clinical interview for DSM-IV-clinician version (SCID-CV) (user's guide interview). American Psychiatric Press, Washington, DC

14. El Missiry A, Sorour A, Sadek A, Fahy T, Abdel Mawgoud M, Asaad T (2004) Homicide and psychiatric illness: an Egyptian study [MD thesis]. Faculty of Medicine, Ain Shams University, Cairo

15. Kay SR, Wolkenfeld F, Murrill LM (1988) Profiles of aggression among psychiatric patients: I. Nature and prevalence. J. of Nerv. Ment. Dis. 176: 539-546
16. Kirkpatrick LA, Feeney BC. (2013): A simple guide to IBM SPSS statistics for version 20.0. Student ed. Belmont, Calif.: Wadsworth, Cengage Learning; 2013.

17. Walsh E, Buchnan A, Fahy T (2002) Violence and schizophrenia: examining the evidence. The British Journal of Psychiatry Jun 180(6):490-495. https:// doi.org/10.1192/bjp.180.6.490

18. Rabie M., Hamdi E., Sabri N., Sedrak A., Khowailed A., Loza N., and Rami H. (2016): Sociodemographic indicators for substance use and abuse in Egypt. J Addiction prevention. 2016:4(1): 8 .

19. Krakowski Ml, Czobor P, Citrome L et al (2006) Atypical antipsychotic agents in the treatment of violent patients with schizophrenia and schizoaffective disorder. Archives of General Psychiatry. 63:622-629

20. Krenek M, Maisto SA. (2013). Life event and treatment outcomes among individuals with substance use disorders: a narrative review. Clin psych rev. $33: 470$

21. Unger JB (2012) The most critical unresolved issues associated with race, ethnicity, culture, and substance use. Subst Use Misuse. Mar 47(4):390-395. https://doi.org/10.3109/10826084.2011.638017

22. Swanson JW, Swartz MS, Essock SM, Osher FC et al (2002) The socialenvironmental context of violent behavior in persons treated for severe mental illness. Am J Public Health 92:1523-1531

23. Alcorn III L., Joshua L. Gowin, Charles E. Green, Alan C. Swan, F. Gerard Moeller, and Scott D. Lane. (2013): Aggression, impulsivity, and psychopathic traits in combined antisocial personality disorder and substance use disorder. The journal of neuropsychiatry and clinical neuroscience. Published Online:1 Jul 2013https://doi.org/10.1176/appi. neuropsych.12030060

24. Zois E., Kortlang N., Vollstädt-Klein S., Lemenager T., Beutel M., Mann K., Fauth-Bühler M. (2014): Decision-making deficits in patients diagnosed with disordered gambling using the Cambridge gambling task: the effects of substance use disorder comorbidity. Brain and Behavior . 2014

25. Kelly J. (2009). Violence in schizophrenia rare in the absence of substance abuse Journal of the American Medical Association JAMA-301:2016-2023

26. Fazel S, Grann M, Carlstrom E, Lichtenstein P, Langstrom N (2009) Risk factors for violent crime in Schizophrenia: a national cohort study of 13,806 patients. Journal of Clinical Psychiatry 70(3):362-369

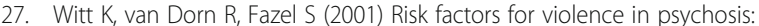
systematic review and meta-regression analysis of 110 studies. Psychiatr. Serv. 52:1358-1366

28. Short T, Thomas S, Mullen P, Ogloff JR. (2013). Comparing violence in schizophrenia patients with and without comorbid substance- use disorders to community controls Acta Psychiatr Scand. Oct;128(4):306-313. doi: https://doi.org/10.1111/acps.12066. Epub 2013 Feb 4.

29. Cornejo C, Simonetti F, Aldunate N, Ibáñez A, López V, Melloni L (2007) Electrophysiological evidence of different interpretive strategies in irony comprehension. Journal of Psycholinguistic Research 36(6):411-430

30. Gray JR, Burgess GC (2004) Personality differences in cognitive control? BAS, processing efficiency, and the prefrontal cortex. Journal of Research in Personality 38:35-36

31. Qiu Y-Q, Tang Y-X, Chan RCK, X-y S, He J (2014) P300 aberration in firstepisode schizophrenia patients: a meta-analysis. PLoS ONE 9(6):e97794. https://doi.org/10.1371/journal. pone. 0097794

32. Simlai J, Nizamie S. H. (1998). Event related potentials-P300, CNV, MRCP in drug naïve and drug free schizophrenia. Thesis submitted to Ranchi University, in partial fulfillment of requirement of degree of doctor of medicine in psychiatry.

33. Chabot RJ, di Michele F, Prichep L (2005) The role of quantitative electroencephalography in child and adolescent psychiatric disorders. Child Adolesc Psychiatr Clin N Am. 14(1):21-53 v-vi

\section{Publisher's Note}

Springer Nature remains neutral with regard to jurisdictional claims in published maps and institutional affiliations. 